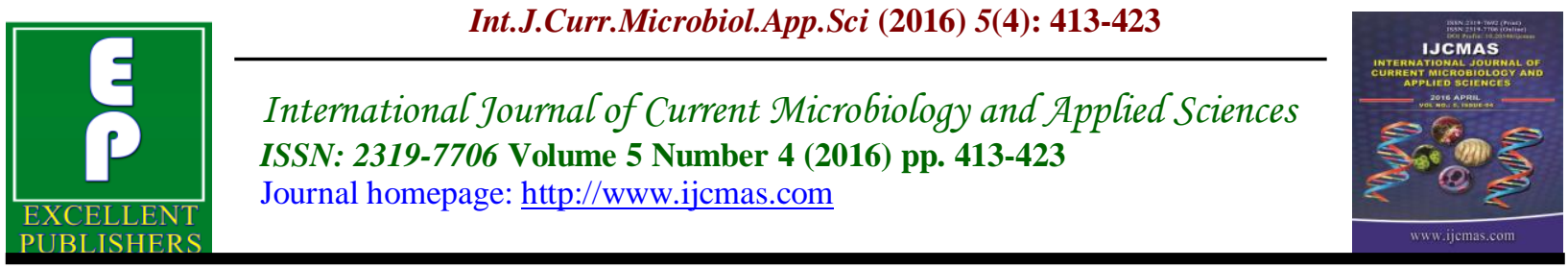

Original Research Article

http://dx.doi.org/10.20546/ijcmas.2016.504.049

\title{
Screening and Isolation of PHB Producers from Plant Sources
}

\author{
Aseem Rajan Wagle, Yogini Manohar Dixit and Babu Vamanrao Vakil*
}

Guru Nanak Institute for Research and Development, Guru Nanak Khalsa College of Arts, Science and Commerce, Matunga, Mumbai 400019, India

*Corresponding author

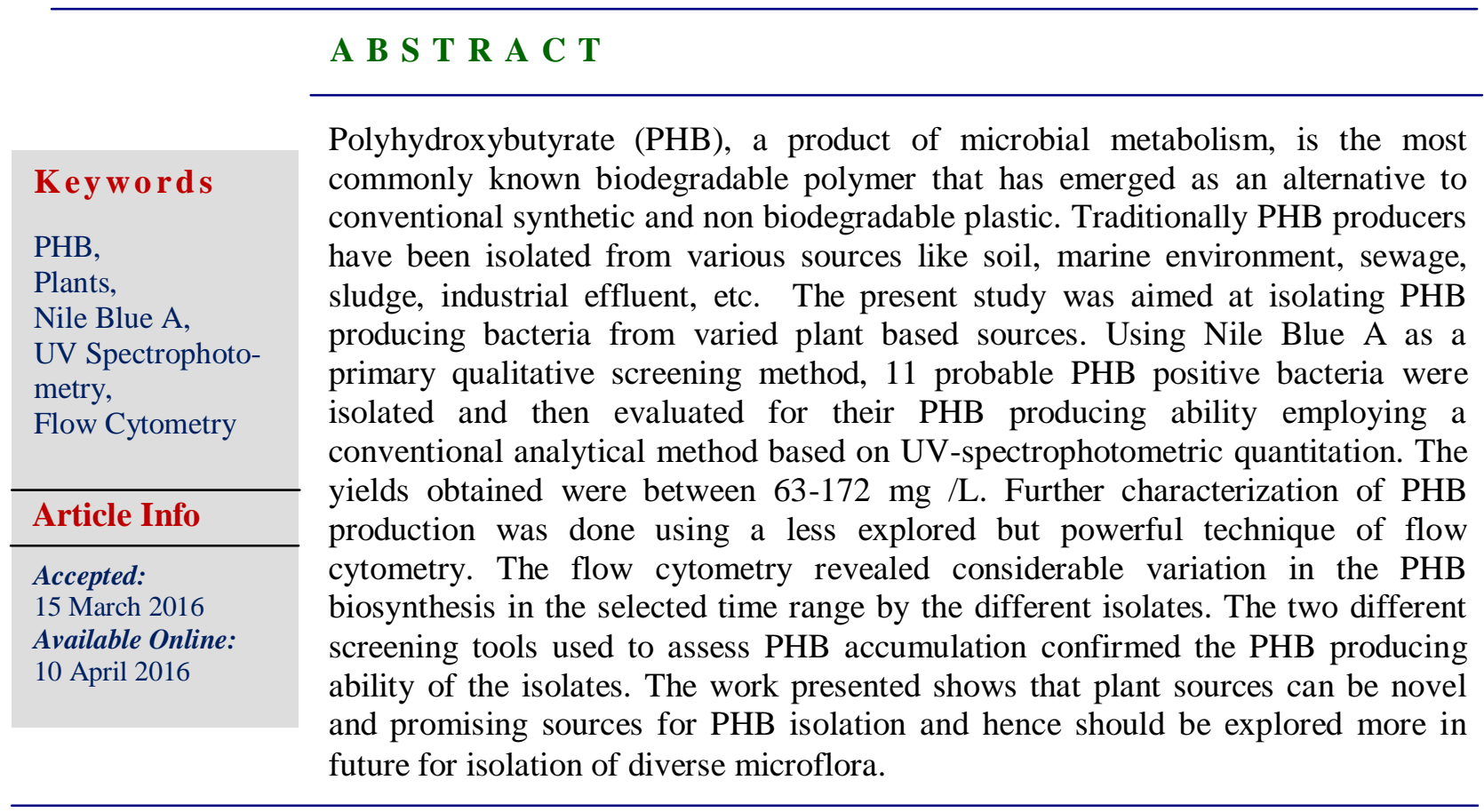

\section{Introduction}

As a result of the problems associated with petrochemical based plastics as regards to its adverse impact on the environment coupled with rapid depletion of fossil fuels, there has been extensive research done on search, production and development of fermentation process to produce biodegradable plastics (Phanse et al., 2011; Chandra \& Rustgi, 1988; Ghanbarzadeh \& Almasi, 2013; Kalia et al., 2000). Polyhydroxyalkanoates (PHA), being biodegradable, can be used as an ecofriendly alternative to the conventionally produced synthetic polymers which are nonbiodegradable and responsible for numerous environmental hazards (Lee, 1996). A wide variety of microbes when cultivated under unfavourable growth conditions, are capable of accumulating PHA as an intracellular reserve food material. The accumulated PHA can be utilized later as a carbon and energy source by the producing bacteria (Nehra et al., 1994). Polyhydroxybutyrate (PHB) is the most common representative of PHA family and is produced by various 
genera such as Azotobacter, Bacillus, Cupriavidus, Pseudomonas, Ralstonia, Rhizobium, etc. (Kumari \& Dhingra, 2013). The conditions favouring PHB production include unbalanced medium composition like higher carbon accumulation, depletion of nitrogen, phosphorous or sulphur and adverse physiological growth conditions like inadequate levels of dissolved oxygen (Kim et al., 1994).

Most researchers have isolated PHB from different environmental sources such as soil (Singh and Parmar, 2011), municipal sewage, sludge (Reddy et al., 2008), marine environments (Arun et al., 2009). However, very less work has been reported with the PHA producing ability of bacteria from the phyllosphere (Kumbhakar et al., 2012).The bacteria are present on plant surface (epiphytes) and/or the interior of plant tissues (endophytes) (Oliveira Costa, 2012). Since each plant is a niche environment and plants are known to produce an array of photochemicals, it is expected to create stressful environment for the bacterial flora possibly resulting in selection of bacteria that could produce PHB. The present study was carried out to screen and isolate PHB producers from varied plant sources like infected plants, parasite invaded host plant and its parasitic plant, root nodules of leguminous plants.

Primary screening of PHB producing bacteria has been based on methods involving use of stains such as Sudan Black $\mathrm{B}$ and more sensitive fluorescent dyes like Nile Blue A (Pierce \& Schroth, 1994) The method is considered as rapid, reliable and reasonably sensitive primary screening and allows detection of PHA producers without affecting their growth (Spiekermann et al., 1999). In the present study the isolates obtained were screened using Nile Blue A staining method and were further evaluated for their PHA producing ability by a simple but specific spectrophotometric assay (Law and Slepecky, 1960) and later by flow cytometry which is a less explored but promising non-invasive method (Shakeri et al., 2011).

\section{Materials and Methods}

Analytical grade chemicals were obtained from Merck Pvt, India and Sigma Aldrich Pvt. Ltd., India while bacteriological media were procured from HI-Media Pvt. Ltd., India. Nile Blue A was procured from Sigma Aldrich Pvt. Ltd., India

\section{Isolation of Probable PHB Producers}

\section{Procurement of Samples}

Host plant leaves (Leaves of Mangifera indica) and its parasitic plant (Leaves of plant belonging to Loranthus genus) was procured from a Mumbai-Goa highway near Dapoli. Infected Alstonia scholaris (L.) R. $\mathrm{Br}$ (Indian Devil Tree) and infected Ficus racemosa (syn. Ficus glomerata Roxb.) (Indian Fig Tree) leaves were obtained from the campus of G.N.Khalsa College, Matunga, Mumbai. Root nodules of Arachis hypogaea (Peanut), Vigna unguiculata (Black eyed peas) and Glycine max (Soyabean) were a gift from Miraj College, Sangli, Maharashtra, India. The samples were put in a sterilized air tight plastic bag and transported to laboratory quickly and stored in the refrigerator till processing which was quickly done within $72 \mathrm{hr}$ of receipt.

\section{Sample Pretreatment and Extraction}

The plant samples were washed thoroughly with sterile water to get rid of superficial dust and particulate matter. Two more washes were given on the laminar flow 
bench and the different parts of the sample were separated using alcohol sterilized scissors. The plant parts were disinfected superficially with the treatment with $70 \%$ alcohol for $30 \mathrm{~s}$, ethanol for $30 \mathrm{~s}, 0.5 \%$ sodium hypochlorite for 2-3 minutes and then 3 rinses in sterile distilled water were given. The cleaned parts were ground using sterile mortar and pestle (Oliveira Costa, 2012; Maroof Ahmed et al., 2012). In case of host and parasitic plant the whole leaves were ground separately using sterile saline to get the slurry. The warts or blisters on the infected plant leaves and the root nodules of leguminous plants were crushed to obtain the exudate. Approximately $5 \mathrm{mg}$ or $\mathrm{ml}$ of crushed sample was dispensed in $250 \mathrm{ml}$ conical flask with $50 \mathrm{ml}$ sterile saline and kept for extraction at $200 \mathrm{rpm} / 37^{\circ} \mathrm{C}$ for $2 \mathrm{~h}$.

\section{Primary Screening}

The supernatants of the above extracts were diluted 10 fold serially, vortexed and surface spread on M9 agar medium (Maniatis, 1982) incorporated with Nile Blue A to a final concentration of $25 \mathrm{ppm}$ (Sujatha et al., 2004). The plates were incubated at $37^{\circ} \mathrm{C}$ up to $48 \mathrm{~h}$ and observed under $366 \mathrm{~nm}$ UV light for detection of fluorescent colonies as potential producers of PHB. All the phenotypically distinct colonies showing fluorescence were transferred onto fresh M9 medium plates with Nile Blue A to confirm the $\mathrm{PHB}$ production by pure single culture growth.

\section{Shake Flask Fermentation for Secondary Screening}

The positive isolates obtained from primary screening were subjected to secondary screening in shake flask fermentation. The cultures were grown in Luria Bertani broth for a period of $16-18 \mathrm{~h}$ at $150 \mathrm{rpm}$ at $37{ }^{\circ} \mathrm{C}$ after then inoculated $(10 \%)$ in $50 \mathrm{ml} \mathrm{M} 9$ broth as the production medium in a $250 \mathrm{ml}$ conical flask and cultivated at $150 \mathrm{rpm} / 37$ ${ }^{\circ} \mathrm{C}$ for $48 \mathrm{hrs}$. The grown cultures were analyzed by two different analytical tools to assess their potential to produce PHB. Samples were withdrawn aseptically at suitable time intervals and analyzed for PHB content.

\section{Sample Preparation and UV Spectrophotometry}

Ten $\mathrm{ml}$ of representative broth sample was withdrawn and centrifuged at 11,500 $\mathrm{g}$ for $10 \mathrm{~min}$. The biomass pellet was then extracted for the PHA polymer content as per the method of John and Ralph, (1961) with slight modifications. The pellet was washed with $10 \mathrm{ml}$ of acetone and ethanol $(1: 1 \mathrm{v} / \mathrm{v})$, centrifuged, resuspended in equal volume of $4 \%$ sodium hypochlorite and incubated at room temperature for $45 \mathrm{~min}$. The whole mixture was recentrifuged and pellet was rewashed as described previously. Finally the washed pellet containing the polymer was dissolved in equal volume of hot chloroform and then filtered using Whatman filter paper No.1.

The filtrate was evaporated to dryness and to the residue, $10 \mathrm{ml}$ of conc. sulphuric acid was added followed by heating in a boiling water bath for $15 \mathrm{~min}$. This step converts the polymer into brown coloured crotonic acid which was cooled and then read spectrophotometrically at $235 \mathrm{~nm}$ against sulfuric acid blank using a SHIMADZU UV 1650 PC system. The PHB content was found out from a standard graph constructed using pure crotonic acid. The concentration of crotonic acid obtained from the extracted broth corresponds to the concentration of PHB. The yield of PHB was calculated as per the formula below: 
yield of $P$ PHB $\left(\frac{m g}{L}\right)=\frac{Y * \text { dilution factor } * 100 \text { fluorescent colonies were seen for the }}{0.924}$ soybean sample, 2 each for peanut, black eyed peas, host plant while 1 each

Where Y: Absorbance of crotonic acid from samples at $235 \mathrm{~nm}$

\section{Sample Preparation and Flow Cytometry}

The probable PHB isolates from primary screening were screened by flow cytometry to better understand the PHB accumulation pattern inside the bacterial cells. The isolates were grown in the manner as mentioned earlier for spectrophotometric analysis. After $24 \mathrm{~h}$ and $48 \mathrm{hr}$ incubation, $1 \mathrm{ml}$ of the culture broth was centrifuged at $11500 \mathrm{~g}$ for $5 \mathrm{~min}$ to obtain pellet. The sample preparation for flow cytometry was carried out as per Shakeri et al. (2011) with minor modifications. The pellet was resuspended in $35 \%$ ethanol and incubated at room temperature for $15 \mathrm{~min}$. This was further centrifuged and the pellet was resuspended in phosphate buffered saline ( $\mathrm{pH}$ 7).To this mixture, Nile Blue A dye was added at a final concentration of $25 \mathrm{ppm}$. After $20 \mathrm{~min}$ incubation at room temperature, the sample was analyzed by flow cytometry. The analysis was performed as per the instruction manual, using an Attune ${ }^{\circledR}$ Acoustic Focusing Cytometer from Applied Biosystems ${ }^{\circledR}$ equipped with an argon laser. The Nile Blue A excitation was done at $488 \mathrm{~nm}$ and emitted fluorescence was measured at $585 \mathrm{~nm}$ in BL2 channel. (User manual, Attune ${ }^{\circledR}$ Acoustic Focusing Cytometer, 2012) Emitted fluorescence intensities of PHB granules for every bacterial sample were compared. One lakh events were analyzed in each sample.

\section{Results and Discussion}

On Nile Blue A primary screening of the varied plant samples, 11 distinct fluorescent colonies were observed. Three different fluorescent colony was observed for Indian Devil Tree and Indian Fig Tree samples. The presence of fluorescent colonies indicated presence of probable PHB producers (Pierce \& Schroth, 1994). Figure 1 a) and b) shows fluorescent PHB colonies from soybean and peanut root nodule samples, respectively. The parasitic plant showed no fluorescent colony which indicates absence of PHB producers.

From the secondary screening of PHB positive isolates using UV spectrophotometric analysis (Table 1), it can be seen that the yield of PHB varies from isolate to isolate, as expected. The PHB yield for the shake flask grown cultures was found to be between $63-172 \mathrm{mg} / \mathrm{l}$. As may be noted, highest PHB production was obtained for isolate PNRN1 which was isolated from the peanut root nodule while least amount was obtained from SYRN1 which was isolated from the soybean root nodule. The isolates SP and UMB2(1) from infected Indian Devil Tree and Indian Fig Tree plant respectively, also produced PHB in good quantity.

The data obtained from the flow cytometric analysis is represented in Figure 2, 3 and 4 in the form of 2 type of 2D scatter plots with one representing SSC side scatter on the Yaxis corresponding to the granulation pattern of the cells vs (BL2) corresponding to fluorescence intensity of the sample on the $\mathrm{X}$-axis and the other representing SSC side scatter vs FSC forward scatter corresponding to cell size (Rieseberg et al., 2001).This analysis was carried out at 3 time points i.e. $0 \mathrm{~h}$-immediately after inoculation of seed and after $24 \mathrm{~h}$ and $48 \mathrm{~h}$ incubation. Thus 6 scatter plots were obtained for selected time points for each isolate. The 
fluorescence intensity of the sample is directly proportional to the PHB production by the cells (Shakeri et al., 2011).The median value of the fluorescence intensity was taken into consideration for interpretation of the data. This represents the multiparametric plot in the form of a single value which helps to compare within heterogeneous populations.

From figure 2 a), b) and c) it can be inferred that for the isolate PNRN1 there was a marked rise in the fluorescence intensity as well as granulation post $24 \mathrm{~h}$ incubation which is indicative of an increase in the BL2 fluorescence intensity along with SSC side scatter. Also from figure $2 \mathrm{~d}$ ), e), and f) the small increase in the FSC forward scatter indicates that there is a slight increase in the cell size during this time interval. The shift in the profile of the 2D scatter plot towards the right and upward direction is a result of the increased production of the PHB polymer over the time period selected. Similar results were obtained for the isolate
UMB2 (1) but there was a steady increase in the profile of the scatter plots with time, signifying consistent PHB build up. Isolate SYRN1 showed a very meager change in the BL2 intensity and a very gradual increase in the FCS forward scatter plots which can be attributed to very low levels of PHB accumulation. Thus for every isolate, when the analysis was carried out at 3 time points, the difference in the fluorescence intensities could be correlated to the changing relative amount of PHB production by the cell population in the culture.

Figure 5 represents the isolates on the basis of their differential median fluorescence intensities after $48 \mathrm{~h}$ incubation, which gives an estimate of the extent of PHB production by the respective culture. Isolate PNRN 1 showed maximum difference in median fluorescence intensity post $48 \mathrm{~h}$ incubation thus indicating highest PHB production whereas SYRN 1 showed least difference indicating low PHB production.

Table.1 Yield of PHB Obtained from UV Spectrophotometric Analysis of the Isolates

\begin{tabular}{|c|c|c|c|}
\hline $\begin{array}{l}\text { Sr. } \\
\text { no. }\end{array}$ & Isolate & Source & $\begin{array}{l}\text { Amount of } \\
\text { PHB (mg/l) }\end{array}$ \\
\hline 1 & HP 1 & \multirow{2}{*}{ Host plant } & 119.81 \\
\hline 2 & HP 2 & & 68.61 \\
\hline 3 & SP & Infected Indian Devil tree & 123.05 \\
\hline 4 & $\operatorname{UMB} 2(1)$ & Infected Indian Fig tree & 125.32 \\
\hline 5 & PNRN 1 & \multirow{2}{*}{ Peanut root nodule } & 172.73 \\
\hline 6 & PNRN 2 & & 101.95 \\
\hline 7 & CWRN 3 & \multirow{2}{*}{$\begin{array}{c}\text { Black eyed peas root } \\
\text { nodule }\end{array}$} & 94.16 \\
\hline 8 & CWRN 4 & & 115.58 \\
\hline 9 & SYRN 1 & \multirow{3}{*}{ Soyabean root nodule } & 63.74 \\
\hline 10 & SYRN2 & & 131.49 \\
\hline 11 & SYRN4 & & 87.99 \\
\hline
\end{tabular}


Figure.1 Fluorescent Colonies Indicating PHB Producers Obtained on M9 agar using Nile Blue A Screening Method

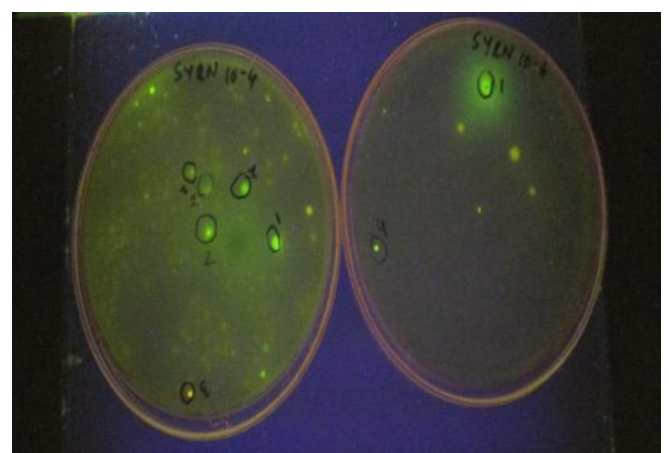

a)Soyabean root nodule sample

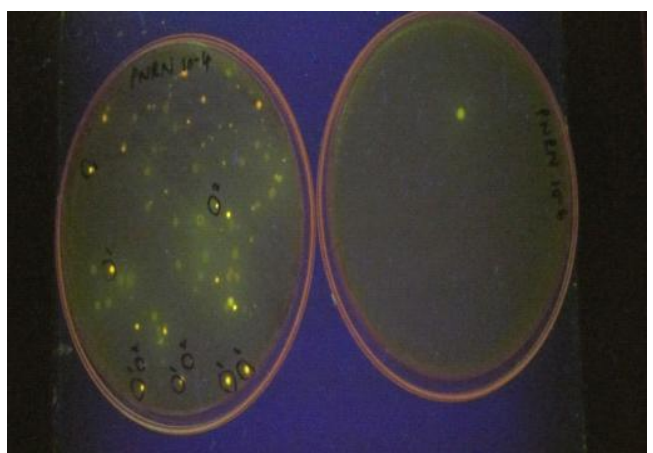

b)Peanut root nodule sample

Figure.2 Scatter Plot for Isolate PNRN1 Obtained from Flow Cytometric Analysis Representing a) SSC vs BL2 at 0h b) SSC vs BL2 at 24h c) SSC vs BL2 at 48h d) SSC vs FSC at $0 \mathrm{~h}$ e) SSC vs FSC at 24h f) SSC vs FSC at $48 \mathrm{~h}$

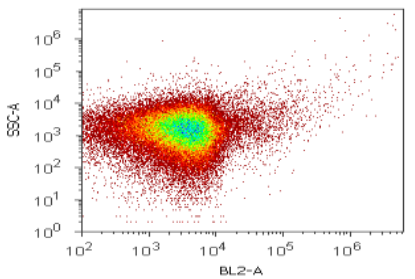

Figure 2 a)

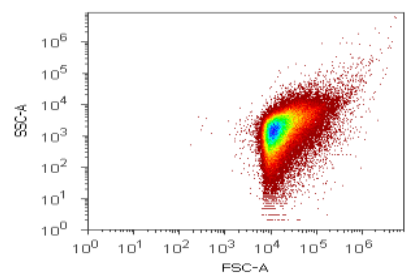

Figure 2 d)

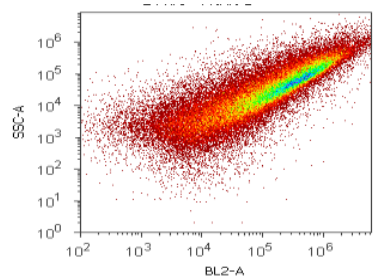

Figure 2 b)

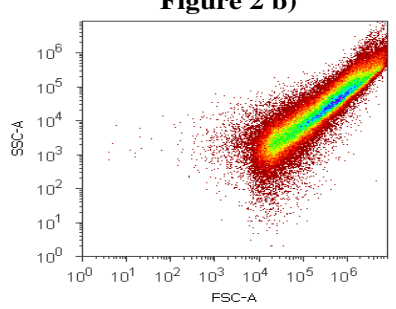

Figure 2 e)

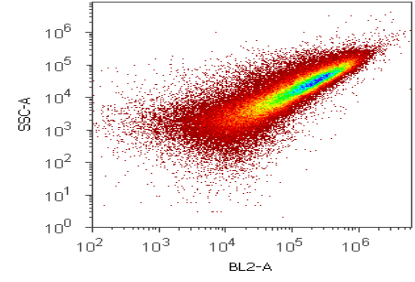

Figure 2 c)

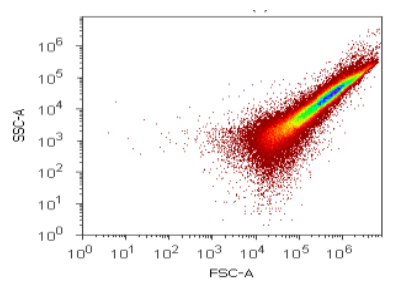

Figure 2 f)

SSC: Side Scatter, BL2: Fluorescence Intensity, FSC: Forward Scatter 
Figure.3 Scatter Plot for Isolate UMB2(1) Obtained from Flow Cytometric Analysis Representing a)SSC vs BL2 at 0h b) SSC vs BL2 at 24h c) SSC vs BL2 at 48h d)SSC vs FSC at $0 \mathrm{~h}$ e) SSC vs FSC at 24h f) SSC vs FSC at $48 \mathrm{~h}$

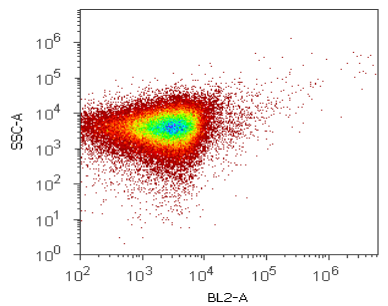

Figure 3 a)

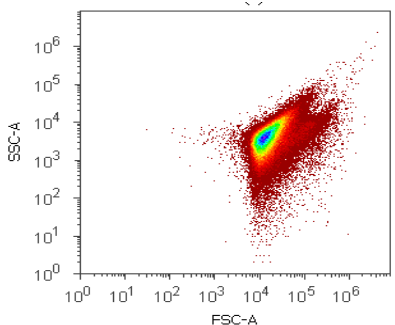

Figure 3 d)

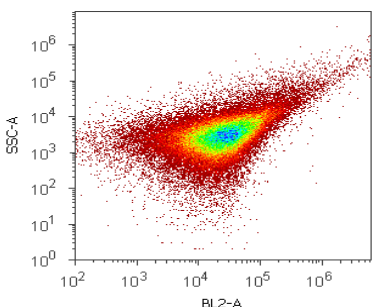

Figure 3 b)

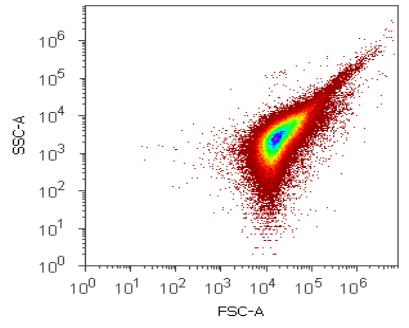

Figure 3 e)

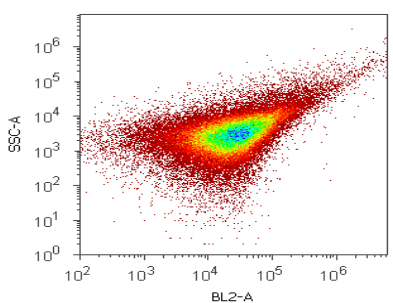

Figure 3 c)

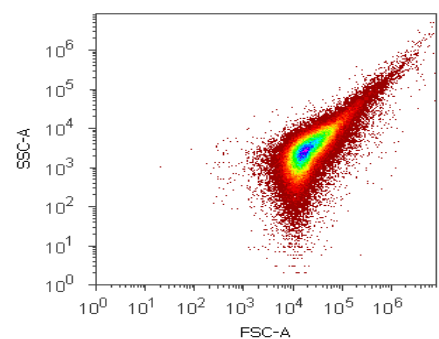

Figure 3 f)

SSC: Side Scatter, BL2: Fluorescence Intensity, FSC: Forward Scatter

Figure.4 Scatter Plot for Isolate SYRN1 Obtained from Flow Cytometric Analysis Representing a)SSC vs BL2 at 0h b) SSC vs BL2 at 24h c) SSC vs BL2 at 48h d)SSC vs FSC at $0 \mathrm{~h}$ e) SSC vs FSC at $24 \mathrm{~h} \mathrm{f)} \mathrm{SSC} \mathrm{vs} \mathrm{FSC} \mathrm{at} 48 \mathrm{~h}$
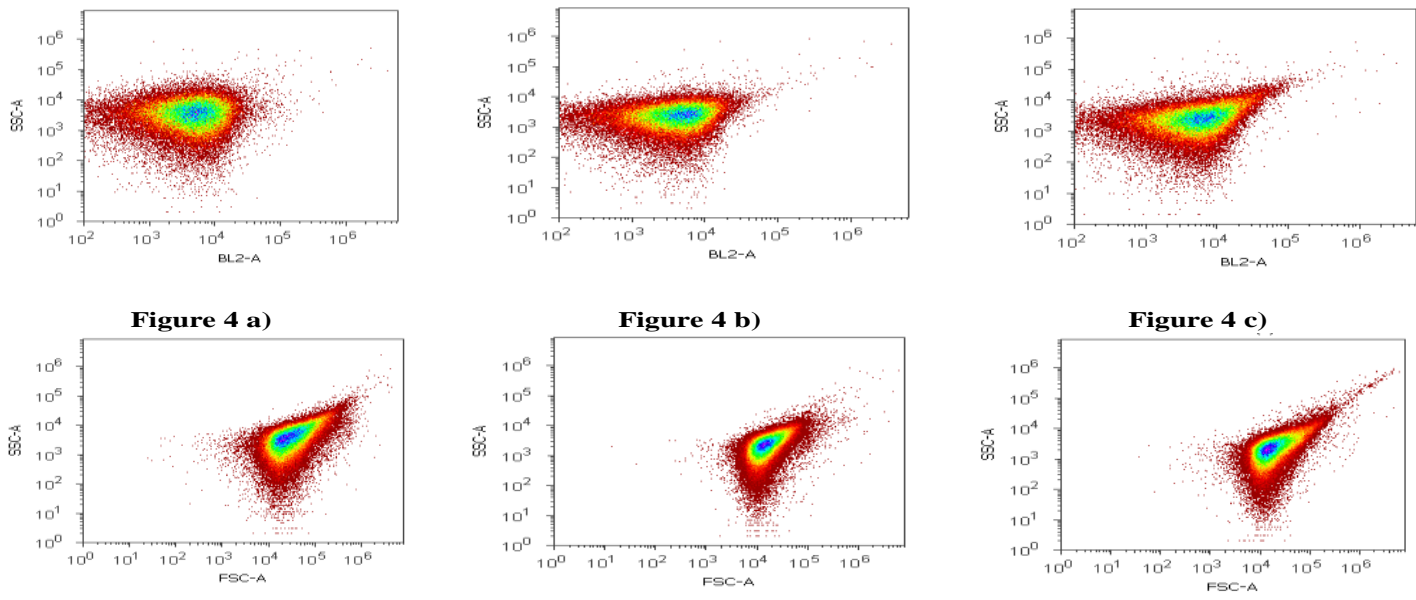

Figure 4 d)

Figure 4 e)

Figure 4 f)

SSC: Side Scatter, BL2: Fluorescence Intensity, FSC: Forward Scatter 
Figure.5 Comparative Differential Median Fluorescence Intensities for PHB Isolates Analyzed by Flow Cytometry

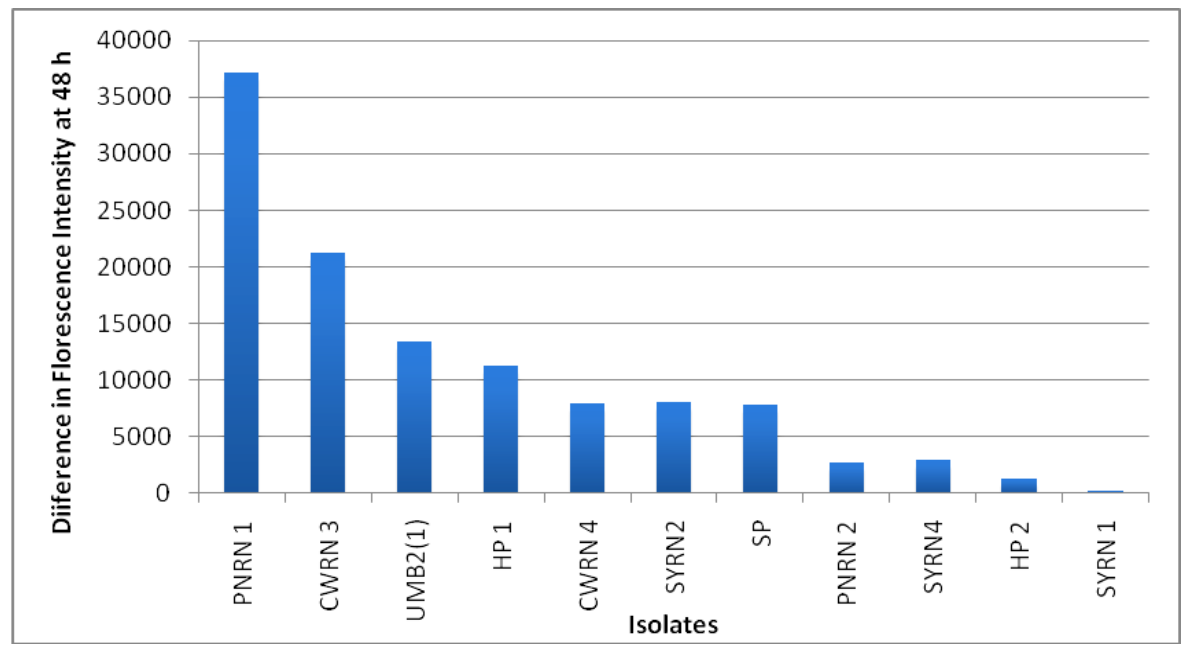

The current need for attention on environmental issues caused by difficulty in petrochemical waste management and fossil fuel exhaustion has given stimulus to research dealing with finding alternatives to conventionally produced synthetic plastics. This aspect has led to an increased appreciation of the role of biodegradable polymers like PHB obtained from microbial sources (Anderson and Dawes, 1960). Many natural habitats have been explored till date to isolate organisms having PHB producing potential but the plant sources still remain a less reported niche. Plant sources provide a complex and unfavorable environment which is also known to be required for PHB production. In the present study, maximum number of probable PHB producers were obtained from the root nodules of leguminous plants through primary screening based on Nile Blue A plate assay. These results are similar to the previous studies by others (Kumbhakar et al., 2012; Laxman \& Shamala, 2003; Mercan et al., 2002). It is noteworthy that in our studies a considerable number of PHB producers was also obtained from novel plant sources involving host-parasite association and infected plant varieties. The present work thus, led to the isolation of reasonably good amount of PHB producers from the novel plant sources. The further study involved evaluating the bacterial isolates for their PHB production. The secondary screening was done by UV spectrophotometric method which provides a rapid, reliable and convenient extraction method to quantify PHB indirectly (Law and Slepecky, 1960). The yields obtained by this method for the given isolates are comparable to reports by Soam et al. (2012) and Muralidharan \& Radha (2014).

Although the flow cytometry technique is commonly used in many clinical applications it has not yet gained popularity for microbial applications particularly quantitation of microbial secondary metabolites (Kacmar et al., 2005; Ratcliff et al., 2008). Hence in the current study, this technique was explored to compare PHB producing capability of various isolates by comparing the difference in the median fluorescence intensities over the period of PHB production. The cultures exhibited diverse pattern of PHB accumulation in the selected time period which can be seen as change in the BL2 fluorescence intensity. 
Also additional valuable data with respect to variation in granularity and cell size within cultures could be studied from the work carried out. Similar work has been reported by Shakeri et al. (2011) who have screened bacteria extensively from heterologous populations for PHB production using the same principle. The advantage of using this technique is that it gave not only useful complementary data to the conventional methods like UV spectrophotometry but also provides additional information to differentiate between varied bacterial populations based on their single cell characteristics like cell size, content and fluorescence intensity measurements simultaneously (Baltz et al., 2010; Rieseberg et al., 2001). Flow cytometry though expensive is a quicker and reliable technique and detects the polymer of interest (PHB) in its native form within the cell without any need to derivatize it unlike UV spectrophotometry. The technique is also more sensitive as it depends on detection of fluorescence. This makes flow cytometry a reliable tool that can be used in combination with the conventional screening tools for quantitation of PHB production.

In conclusion, as an adaptation system to the stressful conditions of nutrient imbalance and adverse physiological environment in the phyllosphere or rhizosphere, a considerable proportion of bacteria were isolated from the selected plant sources and assessed for their capability to accumulate PHB using two analytical tools. Further these isolates can be subjected to medium optimization and varied culture conditions for maximizing PHB production. The highest PHB producers can be identified using classical biochemical tests and molecular biology techniques like $16 \mathrm{~S}$ rRNA. The polymer can also be characterized by using other analytical tools like GC,GC-MS,FTIR and the PHB production process could be scaled up to laboratory fermentor level for increased yield.

\section{Acknowledgement}

The authors are grateful to Prof. Shashikant Kamble, Dept. of Microbiology. Miraj College, Sangli, Maharashtra for providing the root nodules of leguminous plants. The authors also wish to acknowledge Mr.Yogesh Suryavanshi from National Facility for Biopharmaceuticals, G.N.Khalsa College, Mumbai for providing assistance in the flow cytometry work.

\section{References}

Ahmed, M., Hussain, M., Dhar, M., Kaul, S. 2012. Isolation of microbial endophytes from some ethnomedicinal plants of Jammu and Kashmir. Natural Products Plant Res., 2(2): 215-220.

Anderson, A., Dawes, E. 1990. Occurrence, metabolism, metabolic role and industrial uses of bacterial polyhydroxyalkanoates. Microbiol. Mol. Biol. Rev., 54(4): 450-472.

Arun, A., Arthi, R., Shanmugabalaji, V., Eyini, M. 2009. Microbial production of poly- $\beta$-hydroxybutyrate by marine microbes isolated from various marine environments. Biores.Technol., 100(7) : 2320-2323.

Chandra, R., Rustgi, R. 1998. Biodegradable polymers. Progress in polymer science. 23(7): 1273-1335.

Baltz, R., Demain, A., Davies, J. 2010. Manual of industrial microbiology and biotechnology. Third edition. American Society for Microbiology Press. Washington. United States, Pp. 568-69.

Ghanbarzadeh, B., Almasi, H. 2013. Biodegradable polymers. 
Biodegradation-Life of Science. InTech, Rijeka, Croatia, Pp.141-186.

John, H., Ralph, A. 1961. Assay of poly- $\beta$ hydroxybutyric acid. J. Bacteriol., 82: 33-36.

Kalia, V.C., Raizada, N., Sonakya, V. 2000. Bioplastics. J. Sci. Industrial Res., 59(6): 433-445.

Kacmar, J., Carlson, R., Balogh, S., Srienc, F. 2005. Staining and quantification of Poly-3- hydroxybutyrate in Saccharomyces cerevisiae and Cupriavidus necator cell populations using automated flow cytometry. Cytometry, 69: 27-35.

Kim, B., Lee, S., Lee, S., Chang, H., Chang, Y., Woo, S. 1994. Production of poly (3-hydroxybutyric acid) by fed-batch culture of Alcaligenes eutrophus with glucose concentration control. Biotechnol.

Bioengineering, 43(9): 892-898.

Kumari, P., Dhingra, H. 2013. Studied on isolation character of $\mathrm{PHB}$ producing microorganisms isolated from root nodules of leguminous plants. The Bioscan, 8(1) :109-113.

Kumbhakar, S., Singh, P., Vidyarthi, A. S. 2014. Screening of root nodule bacteria for the production of polyhydroxyalkanoate (PHA) and the study of parameters influencing the PHA accumulation. African $J$. Biotechnol., 11(31): 7934-7946.

Lakshman, K., Shamala, R. 2003. Enhanced biosynthesis

of polyhydroxyalkanoates in a mutant strain of Rhizobium meliloti. Biotechnol. Lett., 25(2): 115-119.

Law, J., Slepecky, R. 1961. Assay of poly- $\beta$ hydroxybutyric acid. $J$. Bacteriol., 82(1): 33-36.

Lee, S.Y. 1996. Bacterial polyhydroxy alkanoates. Biotechnol. Bioengineering, 49(1): 1-14.
Maniatis, T. 1982. Molecular Cloning, A Laboratory Manual, First edition. Cold Spring Harbour Publisher, New York, United States, Pp. 68.

Mercan, N., Aslim, B., Yüksekdağ, N., Beyatli, Y. 2002. Production of polyb-hydroxybutyrate (PHB) by some Rhizobium bacteria. Turkish J. Biol., 26(4): 215-219.

Muralidharan, R., Radha, K. 2015. A kinetic study of polyhydroxybutyrate production on nitrogen limited medium using Bacillus subtilis MTCC 9763 through a two stage cultivation strategy. J. Environ. Biol., 36(3): 537.

Nehra, K., Dahiya, N., Lathwal, P., Singh, M. 2015. Influence of nutritional and environmental parameters on PHB production by bacteria isolated from contaminated soils, Int. J. Pharma and BioSci., Int. J. Pharma and BioSci., 6(1): 676-691.

Costa, L., Queiroz, M., Borges, A., Moraes, C., Araújo. 2012. Isolation and characterization of endophytic bacteria isolated from the leaves of the common bean (Phaseolus vulgaris). Brazilian $J$. Microbiol., 43(4): 1562-1575.

Phanse, N., Chincholikar, A., Patel, B., Rathore, P., Vyas, P., Patel, M. 2011. Screening of PHA (poly hydroxyalkanoate) producing bacteria from diverse sources. Int. J. Biosci., 1(6): 27-32.

Pierce, L., Schroth, M. 1994. Detection of Pseudomonas colonies that accumulate poly- $\beta$-hydroxybutyrate on Nile blue medium. Plant Dis., 78(7): 683-685.

Ratcliff, W., Kadam, S., Denison, R. 2008. Poly-3-hydroxybutyrate (PHB) supports survival and reproduction in starving rhizobia. FEMS Microbiol. Ecol., 65(3): 391-399. 
Reddy, S., Thirumala, M., Mahmood, S. 2009. Production of PHB and P (3HBco-3HV) biopolymers by Bacillus megaterium strain OU303A isolated from municipal sewage sludge. World J. Microbiol. Biotechnol., 25(3): 391397.

Rieseberg, M., Kasper, C., Reardon, K.F., Scheper, T. 2001. Flow cytometry in biotechnology. Appl. Microbiol. Biotechnol., 56(3-4): 350-360.

Shakeri, S., Emtiazi, G., Roghanian, R. 2011. Comparison of intracellular polyhydroxybutyrate granules formation between different bacterial cell subpopulations by flow cytometry. Jundishapur $J$. Microbiol., 4(4): 229-238.

Singh, P., Parmar, N. 2013. Isolation and characterization of two novel polyhydroxybutyrate (PHB)producing bacteria. African $J$. Biotechnol., 10(24): 4907-4919.
Soam, A., Singh, A., Singh, R., Shahi, S. 2012. Optimization of culture conditions for bio-polymer producing Bacillus mycoides (WSS2) bacteria from sewage. Curr. Discovery, 1(1): 27-32.

Spiekermann, P., Rehm, B. H., Kalscheuer, R., Baumeister, D., Steinbüchel, A. 1999. A sensitive, viable-colony staining method using Nile red for direct screening of bacteria that accumulate polyhydroxyalkanoic acids and other lipid storage compounds. Arch. Microbiol., 171(2): 73-80.

Sujatha, K., Mahalakshmi, A., Shenbagarathai, R. 2005. A study on accumulation of PHB in native Pseudomonas isolates LDC-5 and LDC-25. Indian J. Biotechnol., 4(2): 216-221.

\section{How to cite this article:}

Aseem Rajan Wagle, Yogini Manohar Dixit and Babu Vamanrao Vakil. 2016. Screening and Isolation of PHB Producers from Plant Sources. Int.J.Curr.Microbiol.App.Sci.5(4): 413-423. doi: http://dx.doi.org/10.20546/ijcmas.2016.504.049 